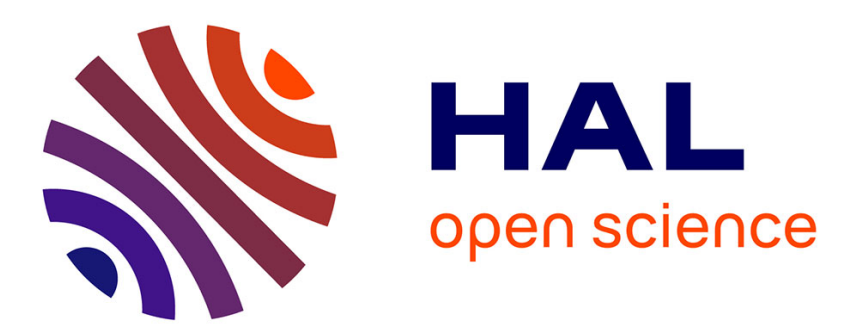

\title{
Application of grey system theory to phosphorite sinter process: From modeling to control
}

Nigina Toktassynova, Hassen Fourati, Batyrbek Suleimenov

\section{To cite this version:}

Nigina Toktassynova, Hassen Fourati, Batyrbek Suleimenov. Application of grey system theory to phosphorite sinter process: From modeling to control. Asian Journal of Control, 2020, 3 (1), pp.13-22. 10.1002/asjc. 2348 . hal-02967731

\section{HAL Id: hal-02967731 \\ https://hal.inria.fr/hal-02967731}

Submitted on 15 Oct 2020

HAL is a multi-disciplinary open access archive for the deposit and dissemination of scientific research documents, whether they are published or not. The documents may come from teaching and research institutions in France or abroad, or from public or private research centers.
L'archive ouverte pluridisciplinaire HAL, est destinée au dépôt et à la diffusion de documents scientifiques de niveau recherche, publiés ou non, émanant des établissements d'enseignement et de recherche français ou étrangers, des laboratoires publics ou privés. 


\title{
Application of grey system theory to phosphorite sinter process: From modeling to control
}

\author{
Nigina Toktassynova ${ }^{1}$ | Hassen Fourati ${ }^{2}$ (D) | Batyrbek Suleimenov ${ }^{1}$
}

${ }^{1}$ Department of Automation and Control, Satbayev University, Kazakhstan

${ }^{2}$ GIPSA-Lab, University Grenoble Alpes, Grenoble, France

\section{Correspondence}

Hassen Fourati, GIPSA-Lab, University Grenoble Alpes, Grenoble 38400, France. Email: hassen.fourati@gipsa-lab.fr

\begin{abstract}
The sintering process of phosphorite ore occurs with a large amount of return caused by untimely process control. The control task of phosphorite ore sintering is to regulate the parameters of the process to obtain a high quality sinter. The parameter clearly responsible for sinter quality is the temperature in the wind box. Therefore, in order to solve the control task, it is necessary to predict the highest temperature of the charge (also known as the burn through point (BTP)). In this paper, the theory of grey systems is used as a predictive model, which makes it possible to obtain an adequate model that uses a small number of initial samples of real temperature data. Based on the grey model $\operatorname{GMC}(1, \mathrm{n})$ a new optimal model is presented, which is constructed by using optimization algorithm. Optimal model predicts the BTP, and to establish an optimal regulation, a control synthesis is carried out through an optimization of the prediction according to the "particle swarm" algorithm.
\end{abstract}

\section{KEYWORDS}

burn through point, control structure, grey system, optimization algorithm, predictive model, sinter process

\section{1 | INTRODUCTION}

Sintering in metallurgy is a thermal process that occurs in a metallurgical charge, composed of ore pellets, concentrates, and fuel (coke). The technological process is shown in Figure 1. It begins with the preparation of the phosphorus ore in predetermined proportions, which includes the following components: phosphorus trifle, coke trifle, and secondary return. This return is the bad quality sinter produced after breaking in yellow phosphorus production. Next, the phosphorus ore is mixed with a cold return and other components in a special drum to form the charge.
This latter is loaded onto the moving grate of the sintering machine and is ignited using natural gas or $\mathrm{CO}_{2}$ under the horn. With the help of wind boxes under the grate, an underpressure is created and a stream of hot gases gradually passes through the layer of $260 \mathrm{~mm}$ in height from the top to the underlying layers, ensuring the burning of coke breeze particles and the formation of sinter. At the end of the sintering machine, the sinter is cooled and crushed, and the poorly sintered charge is sent to the beginning of the process.

Due to the non-linearity of the process, real time quality control is a complex task. In practice, the operator

Abbreviations: BTP, burn through point; GMC, convolution grey model; NN, neural network; GM, grey model; GMC, convolution integral grey model; FGMC, first-pair-of-data convolution integral grey model; IGDMC, interval grey dynamic model with convolution integral; DGDMC, deterministic grey dynamic model with convolution integral; OGDMC, optimized grey dynamic model with convolution integral. 
FIGURE 1 Technological chain of the sintering process [Color figure can be viewed at wileyonlinelibrary.com]

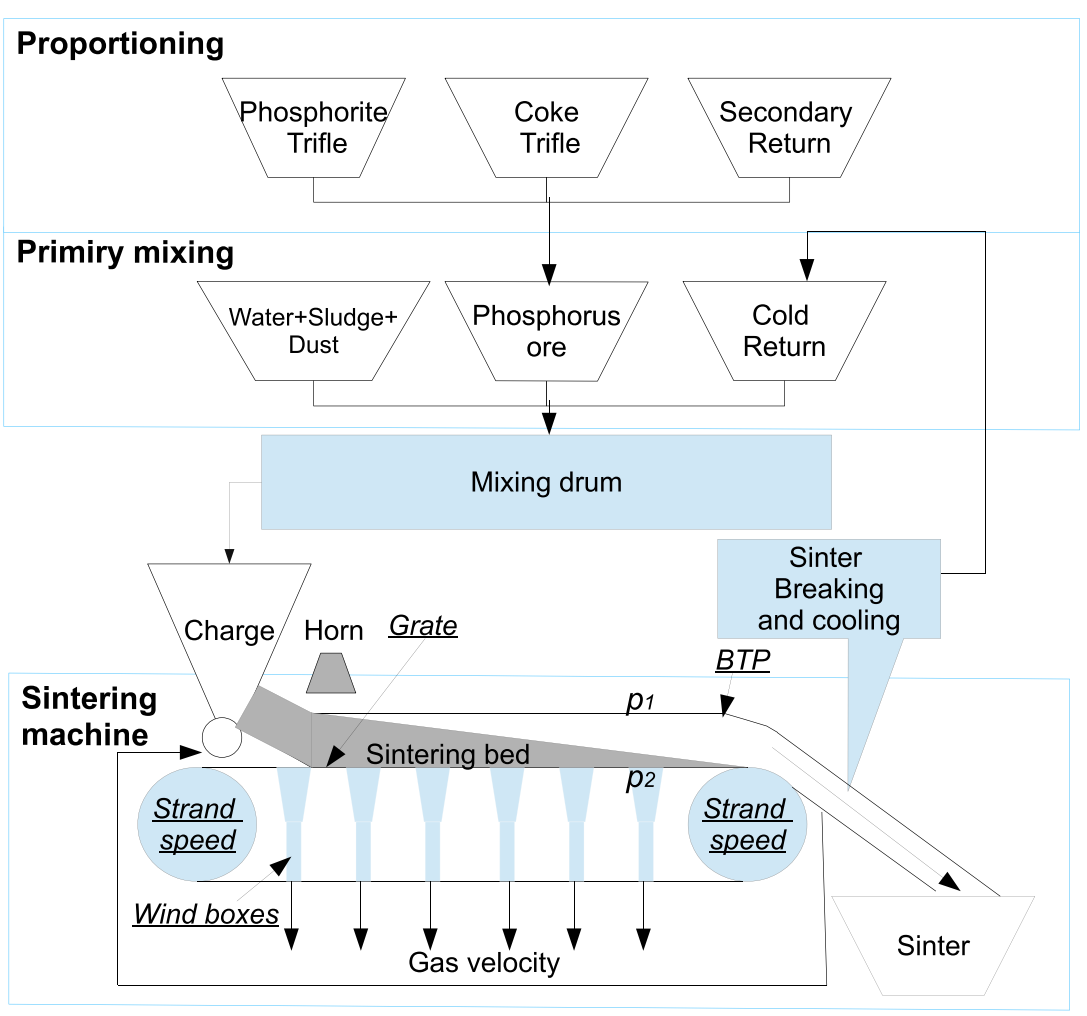

makes changes to the process after the product is obtained, which leads to a large quantity of return and expenses for re-sintering. In this regard, it becomes necessary to predict the sinter quality in advance and control the process based on a predictive model. One of the key indicators of sintering quality is the BTP that indicates the completion of the sintering process and represents the point with the highest temperature. The problem of BTP prediction and control is studied in many works using various algorithms, such as, neural networks (NN), genetic algorithms, cluster analysis, and fuzzy control. Due to the evolution and wide spread of NN, a large number of works related to prediction use this method in BTP prediction and control. NN is used in [1], where the strand speed and BTP at the previous instants of time are given to the network inputs. The network, together with the training algorithm, update the BTP model. In [2], the current value of BTP, temperatures in the middle and end of the process, and the strand speed are inputs to the NN to predict the BTP, and an intelligent control of the iron ore sintering process is proposed. A fuzzy NN is used in BTP prediction [3]. Temperatures in wind boxes are chosen as inputs and the BTP temperature as an output of the model.

Other works [4,5] use two BTP predictive models: temporary and technological. Then, to predict the permeability of lead-zinc ore in [4] the temporary $\mathrm{NN}$ is supplied with 6 previous values for permeability, and the technological $\mathrm{NN}$ uses the temperature of fire, humidity, sulfur, lead, silicon dioxide content, and strand speed. In [5], the BTP grey model $\operatorname{GM}(1,1)$ of lead-zinc ore is applied, and the charge permeability and strand speed of the pallets are fed to the technological neural network input. The approach proposed in [6] for BTP prediction of iron ore sintering uses the integration of grey model and NN. Here the result of the grey model $\operatorname{GM}(1,1)$, especially the gas temperature in wind boxes, is one of the inputs of the back propagation $\mathrm{NN}$, along with the strand speed and BTP at current time. The three works, with two predictive models, use a fuzzy expert controller that maintains the BTP desired position within the specified boundaries.

In [7], a carbon efficiency optimization model and BTP expert-fuzzy controller are presented. The main idea is to improve carbon efficiency by using an intelligent integrated control strategy for the BTP. The strand velocity of the carbon efficiency optimization model and BTP expert-fuzzy controller are integrated using the fuzzy satisfaction degree method, which calculates the end strand velocity. The BTP prediction model developed in [8] also uses 2 models. Temperatures in wind boxes are supplied to a BTP soft-sensor model (1st model) to predict the BTP position. The BTP temperature prediction model (2nd model) uses BTP temperatures, obtained in previous steps. The outputs of 2 models together with strand velocity are used as inputs of fuzzy robust control, which is based on an improved Takagi-Sugeno model.

Taking another perspective, genetic algorithms are used within this framework. Thus, in [9], an adaptive genetic algorithm was used to control BTP in the iron ore sintering 
process, where the input layer of the $\mathrm{NN}$ is parameters of the initial material, density, strand speed, and ignition temperature, and the output layer is the values of temperature and underpressure of sintering gases and gases in wind boxes. The genetic NN was used also for the BTP prediction of iron ore sintering in [10], where 707 groups of data were given to the input after clustering and classification of temperature and underpressure vectors from 18 wind boxes. The adaptive structural clustering system is based on a spatial clustering of initial data, a self-organizing NN map for extracting data relevancy properties, and a Kohonen map for the learning network. The idea of clustering is used in [11] for the BTP prediction model of iron ore sintering. The K-means clustering module, the inputs of which are the cold-charge permeability model, the ignition temperature, and the coke residue values, is used to form clusters. The predictive model based on the clusters from K-means module were fed to the dynamic model of temperature and underpressure, which is constructed using the novel genetic programming. A firework algorithm based on a genetic algorithm was proposed in [12] to optimize the sintering terminal prediction model of the support vector machine. Seven main parameters were used as inputs to predict the BTP.

Models that do not use the NN for BTP prediction are based on equations of temperature or BTP. In [13], the authors used a curve fitting method and represent results using a cubic and fifth-order curves for the part near to the BTP position. The authors in [14] describe a two-level hierarchical control system of the BTP and the vertical sintering rate in the iron ore sintering process, where the BTP model, represented by a piecewise-quadratic temperature, depends on the position on the strand. In [15], the BTP can be approximated using a quadratic function of exhaust-gas temperature. A dynamic state space model is proposed to obtain an exhaust-gas temperature, with 6 inputs. A grid search algorithm optimizes parameters for the best combination in a certain range using an evaluation function. In [16], a BTP control in iron ore sintering process is divided into 2 parts: a closed-loop identification model and a generalized predictive control one. In the first part, the BTP is found based on the dynamic autoregressive exogenous model, to calculate which strand speed, amount of moisture, height of the charge, air volume, and underpressure are used. The closed-system identification method is used to dynamically determine the model parameters. The state of BTP also is predicted using a particle swarm optimization algorithm (PSO) [17], which include 4 influence parameters: suction pressure, air input, velocity of sintering machine, and ignition temperature. Obviously, the current BTP is also affected by its previous stage and [18] carried out time series trend analysis for the BTP, which gives the opportunity to determine global and local trend feature variables. These variables are supplied as inputs of a fuzzy controller, constructed on the basis of an operator's experience. The above review of the literature means we can conclude the following drawbacks. First, the prediction of the BTP is based on many different parameters: data of vertical sintering rate, underpressure, permeability, height of the charge, temperature in wind boxes or sintering gases, strand speed and previous values of the BTP. Second, the high accuracy of predictive models is achieved by using a large initial sample or historical data.

Application of existing models for BTP prediction has the following drawbacks. In practice, due to a lack of automation, only the main process parameters are frequently measured. The values of permeability, volume of sintering gases, and properties of charge are obtained only by laboratory experiments. Therefore, it becomes necessary to construct a predictive model using the main measured process parameters. Obtaining a large initial sample is also difficult in terms of developing a prediction model while in production, and it requires a long time to collect and accumulate data to improve prediction accuracy. In this case, if certain conditions of the process change, it will be necessary to collect data and retrain the model. Therefore, it is necessary to use a predictive model that will be adequate for training on a small number of initial samples and will be built in real time. To solve this problem the theory of grey systems developed by Deng in 1982 is used, which is focused on solving prediction problems with a small volume of the original samples and with poor information. The idea of grey systems theory is to consider the process as a "generalized energy system", and emphasizes that non-negative smooth discrete functions can be transformed into a sequence having the approximate exponential law, that is, the so-called grey exponential law [19]. The application of grey models is considered in $[5,6]$ for BTP prediction. In these studies, the $\operatorname{GM}(1,1)$ model is used, in which only the previous values of the predicted variable influence the subsequent prediction. The disadvantage of this approach is the lack of consideration of influencing factors that can improve the accuracy of the prediction model and results.

Therefore, in this paper, a convolution integral grey model $\operatorname{GMC}(1, \mathrm{n})$ is used [20], which allows us to take into account (n-1) influencing factors on the prediction of BTP. Since the model takes into account not only temperature, but also underpressure in wind boxes, the control task is based on changing two variables, the strand speed (standard approach) and the under-pressure. The main idea of this paper is focused on the development of a BTP prediction system based on a small amount of an initial sample to implement it in a future a cheap control system of sinter quality. To achieve this goal the following steps are proposed: (1) determination of parameters that influ- 
ence BTP and can be measured in real-time; (2) analysis of BTP prediction models based on grey system theory; (3) development of optimal BTP prediction model by using a particle swarm optimization algorithm; (4) proposition of control structure of BTP based on forecast optimization method.

The paper is organized as follows. Section 2 presents the sintering process and the samples of the main parameters used for the prediction process. Section 3 is devoted to the grey predictive models, determination of the most accurate model to predict BTP, and development of an algorithm to improve $\operatorname{GMC}(1, \mathrm{n})$. Section 4 presents the structure of the sintering process control based on a predictive optimization. Section 5 provides some concluding remarks.

\section{SINTERING PROCESS AND MAIN PARAMETERS FOR PREDICTION}

The phosphorite ore sintering process takes place in the Novozhambul phosphorus plant, which is located in the south of Kazakhstan, near the Karatau phosphor field. Sintering machine capacity reaches only $50-60 \%$, since the control is performed with a time delay that relies on an operator's decision. The sintering process includes a lot of parameters, which influence BTP. Some of these can be used and measured only through laboratory studies, such as: initial charge composition, pellet size, ignition process parameters, coke content in charge, percent of sinter return and its characteristics; other parameters can be measured in real-time processing: temperature of exhaust-gases in wind boxes, pressure drop across the bed data, strand velocity, sinter height; and finally there are parameters that are calculated or saved in a database, such as previous BTP data. In this paper the temperature in wind boxes, which can be measured in real-time and gas velocity, which can be calculated through a pressure drop across the bed, are used. Sinter height is constant value in production. Temperature curves (Figure 2) are samples of data taken at different times from the process by experiment using thermocouples, under different initial conditions, but for a constant speed, so they are shown only until the desired temperature is reached in order to determine the duration of the process.

According to the literature, gas velocity has an influence on the charge temperature and could be determined through the pressure drop across the bed data $\Delta p$, then the gas velocity $v$ (Figure 3 ) in the lower part of the charge can be calculated using an Ergun equation:

$$
\frac{\Delta p}{L}=\frac{150 \mu \nu(1-\varepsilon)^{2}}{d^{2} \varepsilon^{3}}+\frac{1.75 \rho \nu^{2}(1-\varepsilon)}{d \varepsilon^{3}}
$$

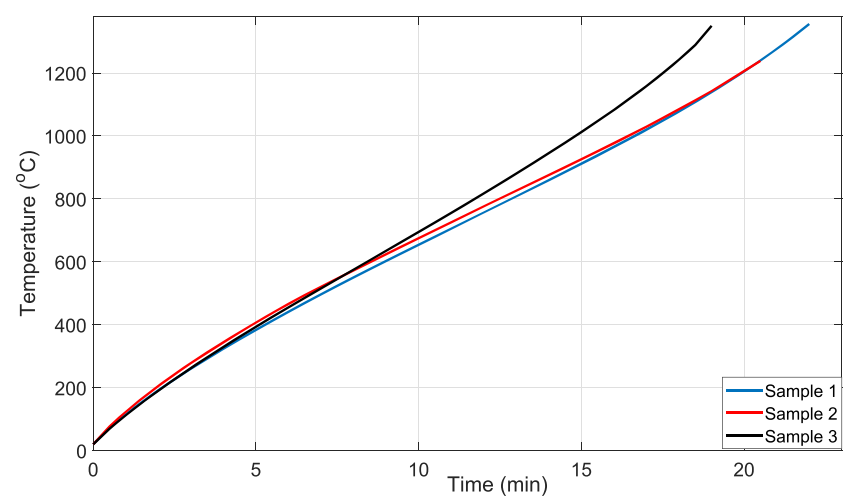

FIGURE 2 Temperature of 3 samples in wind boxes [Color figure can be viewed at wileyonlinelibrary.com]

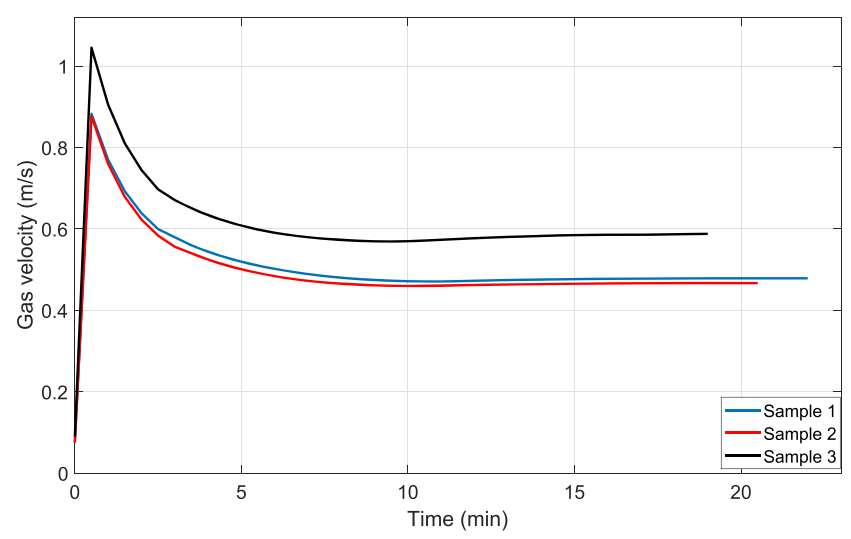

FIGURE 3 Gas velocity of 3 samples in wind boxes [Color figure can be viewed at wileyonlinelibrary.com]

where $L[\mathrm{~m}]$ is the height of the bed, $\mu[\mathrm{Pa} \cdot \mathrm{s}]$ is the dynamic viscosity of gas, $\rho$ is the density of the charge, $\varepsilon$ is the porosity of porous matrix calculated following [21], and $d[m]$ is the equivalent particle size.

\section{3 | DETERMINATION OF BTP GREY MODEL}

Building a data acquisition system with data storage, analysis, and continuous retraining requires tremendous resources both of time and money. Also, the initial data collection, training, and building of an effective system requires constant interruption on the main production process, which impacts its effectiveness. In the production considered for this study there is no data acquisition system, therefore, to obtain data, additional measurement systems were required that were not included in the main control loop. In such conditions, it becomes necessary to use models that require a small amount of the initial sample to train and construct a predictive model. The theory of grey systems satisfies these requirements. 
Grey theory is a relatively new theory for prediction issues. One of the main characteristics of grey system theory is the accumulated generating operation (AGO), which is used to reduce the randomness of the data. The mathematical relation of first-order AGO data (1-AGO) is described as following:

$$
X^{(1)}(t)=\sum_{l=1}^{t} X^{(0)}(l), Y^{(1)}(t)=\sum_{l=1}^{t} Y^{(0)}(l),
$$

where $X^{(0)}, Y^{(0)}$ are the original series and $X^{(1)}, Y^{(1)}$ are 1-AGO data.

The temperature in the wind boxes is considered as predicted series $Y$. The gas velocity $v$, calculated from the known pressure $\Delta p$ (from (1)), which is measured in real time, was selected as an influencing factor $X$.

The influence of several factors on the predicted value for grey models can be considered only on the basis of model modifications $\operatorname{GM}(1, \mathrm{n})$, which is clearly demonstrated in [22]. A continuous convolution integral grey model GMC $(1, \mathrm{n})[21]$ is one of the basic models with $(n-1)$ influencing factors. On the basis of this model, other continuous linear grey models with $(n-1)$ influencing factors were developed. For example, an interval model with a convolution integral $\operatorname{IGDMC}(1, \mathrm{n})[22]$ is intended for prediction of the interval of the variable. Model $\operatorname{FGMC}(1, \mathrm{n})$ [23] is developed on the basis of effectiveness of the first pair of original data. The deterministic grey model with the convolution integral $\operatorname{DGDMC}(1, \mathrm{n})[24]$ distinguishes from $\operatorname{GMC}(1, \mathrm{n})$ by the estimation of first derivative and parameters: first derivative is estimated numerically by the cubic curve of the spline and the parameters of the model, according to the scheme of deterministic convergence. The error in the strength prediction considered by Tien is $0.54 \%$ for $\operatorname{FGMC}(1, \mathrm{n}), 1.25 \%$ for $\operatorname{GMC}(1, \mathrm{n}), 1.85 \%$ for $\operatorname{DGDMC}(1, \mathrm{n})$, and $2.4 \%$ for $\operatorname{IGDMC}(1, \mathrm{n})$. Later in [25], the optimized model $\operatorname{GDMC}(1, \mathrm{n})[23]-\operatorname{OGDMC}(1, \mathrm{n})$ is shown, in which the grey derivative value $d X_{1}^{(1)}(t) / d t$ is determined not by the weighted mean value $X_{1}^{(1)}(t)$ and $X_{1}^{(1)}(t-1)$, but through the weight coefficient $\rho_{i}$, determined using the PSO algorithm [26]:

$$
\rho_{i} X_{i}^{(1)}(t)+\left(1-\rho_{i}\right) X_{i}^{(1)}(t)
$$

Here, a value for the root mean squared percentage error to the priori sample period (RMSPEPR) in the $\operatorname{OGDMC}(1, \mathrm{n})$ decreased by 3.8 times - from $7.07 \%$ to $1.86 \%$. To select the most accurate model for predicting the BTP in the wind box, we will conduct experiments using models $\operatorname{FGMC}(1, \mathrm{n}), \operatorname{GMC}(1, \mathrm{n})$ and $\operatorname{OGDMC}(1, \mathrm{n})$, which have the lowest prediction error value.

\section{1 | GMC(1,n)}

The gas velocity, calculated from the known pressure $\Delta p$, which is measured in real time was selected as an influencing factor. Convolution integral grey model $\operatorname{GMC}(1,2)$ with one influencing factor is a linear differential model:

$$
\frac{d Y^{(1)}(t)}{d t}+b_{1} Y^{(1)}(t)=b_{2} X^{(1)}(t)+u
$$

The grey derivative for the first-order AGO data in Equation (4) is conventionally represented as:

$$
\begin{aligned}
\frac{d Y^{(1)}(t)}{d t} & =\lim _{\Delta t \rightarrow \infty} \frac{Y^{(1)}(t+\Delta t)-Y^{(1)}(t)}{\Delta t} \\
& =Y^{(1)}(t+\Delta t)-Y^{(1)}(t)
\end{aligned}
$$

when $\Delta t \rightarrow 1$. The parameters of Equation (4) are determined by least squares method:

$$
\left[b_{1}, b_{2}, u\right]^{T}=\left(B^{T} B\right)^{-1} B^{T} Y_{R}
$$

where $t$ changes from 1 to $r$ which is the number of initial sample to construct the model in Equation (4),

$$
B=\left[\begin{array}{lll}
-0.5 W_{Y}(1) & 0.5 W_{X}(1) & 1 \\
-0.5 W_{Y}(2) & 0.5 W_{X}(2) & 1 \\
-0.5 W_{Y}(r-1) & 0.5 W_{X}(r-1) & 1
\end{array}\right]
$$

where

$$
\begin{gathered}
W_{Y}(r)=\left(Y^{(1)}(r)+Y^{(1)}(r+1)\right) \\
W_{X}(r)=\left(X^{(1)}(r)+X^{(1)}(r+1)\right) \\
Y_{R}=\left[Y^{(1)}(2), Y^{(1)}(3), \ldots, Y^{(1)}(r)\right]^{T}
\end{gathered}
$$

Prediction of the sinter temperature $\hat{Y}^{(0)}$ is:

$$
\hat{Y}^{(0)}(t)=\hat{Y}^{(1)}(t)-\hat{Y}^{(1)}(t-1)
$$

where $\hat{Y}^{(1)}(t)=Y^{(0)}(1) e^{-b_{1}(t-1)}+\frac{1}{2} e^{-b_{1}(t-1)} \times\left(b_{2} X^{(1)}(t)+u\right)+$ $\frac{1}{2}\left(b_{2} X^{(1)}(t)+u\right)+\sum_{i=2}^{t-1} e^{-b_{1}(t-i)}\left(b_{2} X^{(1)}(i)+u\right)$

\section{2 | FGMC(1,n)}

The differential equation of grey prediction model $\operatorname{FGMC}(1, n)$ presented in $[23]$ is the same as for $\operatorname{GMC}(1, n)$, but modeled with data including the information from the first pair of original data. The parameters of Equation (4) are determined using the LSM Equation (6), where 


$$
\begin{gathered}
B=\left[\begin{array}{lll}
-0.5 W_{Y}(0) & 0.5 W_{X}(0) & 1 \\
-0.5 W_{Y}(1) & 0.5 W_{X}(1) & 1 \\
-0.5 W_{Y}(r-1) & 0.5 W_{X}(r-1) & 1
\end{array}\right] \\
Y_{R}=\left[Y^{(1)}(1), Y^{(1)}(2), \ldots, Y^{(1)}(r)\right]^{T}
\end{gathered}
$$

Prediction of the sinter temperature is:

$$
\begin{aligned}
\hat{Y}^{(0)}(t)= & Y^{(0)}(0) e^{-b_{1} t}+u(t-1) \times \\
& \times \sum_{i=1}^{t}\left(\frac{1}{2} e^{-b_{1}(t-i+0.5)} b_{2}\left(X^{(1)}(i)-X^{(1)}(i-1)\right)\right)
\end{aligned}
$$

\section{3 | OGDMC(1,n)}

Differential equation of the Grey dynamic model with convolution integral is:

$\frac{d Y^{(1)}(t)}{d t}+b_{1} Y^{(1)}(t)=b_{2}+\sum_{i=2}^{n}\left(b_{2 i-1} \frac{d X^{(1)}(t)}{d t}+b_{2 i} X^{(1)}(t)\right)$

The parameters of Equation (13) are determined using the LSM Equation (6), the vector $Y_{R}$ is calculated according to Equation (11) and

$$
B=\left[\begin{array}{llll}
-0.5 W_{Y}(1) & 1 X^{(0)}(1) & 0.5 W_{X}(1) \\
-0.5 W_{Y}(2) & 1 X^{(0)}(2) & 0.5 W_{X}(2) \\
& . & \cdot & \cdot \\
-0.5 W_{Y}(r-1) & 1 X^{(0)}(r) & 0.5 W_{X}(r-1)
\end{array}\right]
$$

Prediction of the temperature is determined by Equation (9), where:

$$
\begin{aligned}
\hat{Y}^{(1)}(t)= & Y^{(0)}(1) e^{-b_{1}(t-1)}+u(t-2) \times \\
& \times \sum_{\tau=2}^{t}\left\{\frac{1}{2} e^{-b_{1}\left(t+0.5-\tau+0.5 \lambda_{1}\right)} \times[0.5(f(\tau)+f(\tau-1))+\right. \\
+ & \left.0.5 \lambda_{1}(f(\tau)+f(\tau-1))\right]+\frac{1}{2} e^{-b_{1}\left(t+0.5-\tau+0.5 \lambda_{2}\right)} \times \\
& \left.\times\left[0.5(f(\tau)+f(\tau-1))+0.5 \lambda_{2}(f(\tau)+f(\tau-1))\right]\right\}
\end{aligned}
$$

where $\lambda_{1}=-1 / \sqrt{3}, \lambda_{2}=1 / \sqrt{3}$ and $f(t)=b_{2}+$ $\sum_{i=2}^{n}\left(b_{2 i-1} X^{(0)}(t)+b_{2 i} X^{(1)}(t)\right)$

\section{4 | Size of samples to construct the model}

The number of data to construct a predictive model is an important parameter, which leads to reduction in errors in temperature prediction. Also it allows for the definition of wind boxes, where thermocouples should be installed in the future and reduce their quantity for economy. The main feature of grey theory is its capability to use as few as $n+3$ pairs of data by $\operatorname{GMC}(1, n)[20]$. For each sample, the

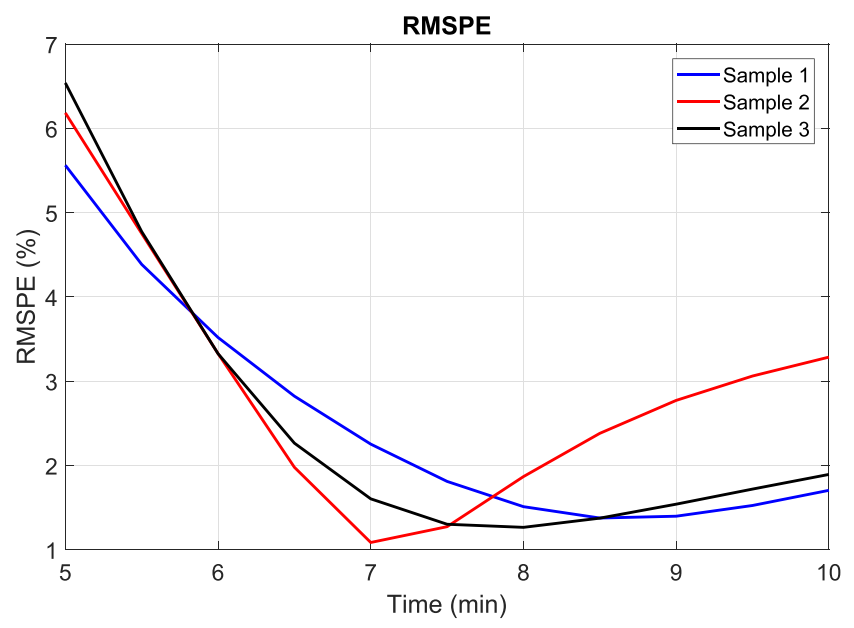

FIGURE 4 Predictive errors for different sample sizes [Color figure can be viewed at wileyonlinelibrary.com]

following experiments were performed: (1) based on the n-pair of series from the sample, the model was built; (2) based on the model, the prediction of the remaining values was made; (3) the prediction results were compared to the original samples and the Root Mean Squared Percentage Error (RMSPE) according to Equation (16) was calculated.

$$
R M S P E=\sqrt{\frac{1}{n} \sum_{i} \frac{\left(\hat{Y}_{i}-Y_{i}\right)^{2}}{Y_{i}^{2}}} \times 100 \%
$$

During the experiments, 17 models were built for each sample ranging from 5 to 20 pair of series. The results of the predicted errors for each sample are presented in Figure 4. The predicted errors for each sample take the minimum values for different pairs of series, ranging from 14 to 17 . The mean smallest predictive error for 3 samples is achieved with 15 pair of series.

\section{5 | BTP prediction results for considered models}

The RMSPE for the post sample period of predictive models for 3 samples (Figure 2) are presented in the Table 1, where the volume of initial sample $r$ to construct the model includes data from the beginning of the sintering process up to $7.5 \mathrm{~min}$. The results of predictive models for sample 1 are shown in Figure 5 and the RMSPE of all samples are presented in Table 1. The best result for predicting the BTP of the charge was obtained as a result of using the $\operatorname{GMC}(1, \mathrm{n})$ model. The predictive results based on grey systems do not improve accuracy in comparison with the other models, but allow the building of an adequate prediction model of BTP in the absence of a large amount of historical temperature data. Additional advantage is the time saving in relation to data collection and model training. 
TABLE 1 RMSPE of grey models, $\%$

\begin{tabular}{|llll} 
No of sample & $\boldsymbol{G M C}(\mathbf{1 , n})$ & $\boldsymbol{F G M C}(\mathbf{1 , n})$ & OGDMC(1,n) \\
\hline 1 & 2.2413 & 4.1062 & 2.6575 \\
2 & 1.6697 & 3.4544 & 3.6921 \\
3 & 1.0952 & 3.8123 & 4.1833 \\
\hline
\end{tabular}

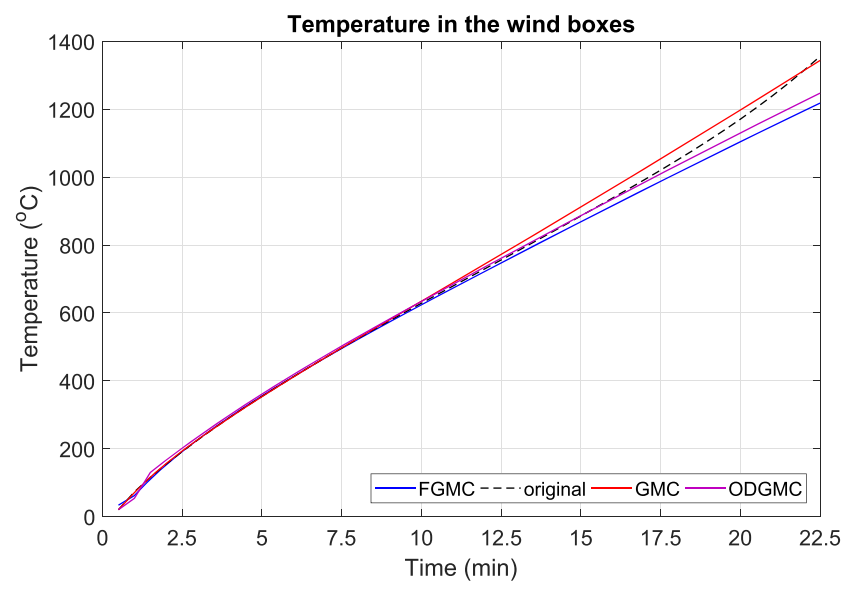

FIGURE 5 Modeling results of sample 1 [Color figure can be viewed at wileyonlinelibrary.com]

TABLE 2 Results of optimal GMC(1,n) model

\begin{tabular}{|c|c|c|c|c|c|}
\hline No & Coef & $\begin{array}{c}\operatorname{GMC}(1, \mathbf{n}) \\
\rho\end{array}$ & $\begin{array}{c}\text { RMSPE } \\
\rho, \%\end{array}$ & $\begin{array}{c}\operatorname{GMC}(1, \mathbf{n}) \\
\rho_{i}\end{array}$ & $\begin{array}{c}\text { RMSPE } \\
\rho_{i}, \%\end{array}$ \\
\hline & $\rho$ & 0.2261 & & $0 \& 0.2283$ & \\
\hline \multirow[t]{3}{*}{1} & $b_{1}$ & -0.0005 & 1.2120 & -0.0005 & 1.2110 \\
\hline & $b_{2}$ & 56.3769 & & 56.3554 & \\
\hline & $u$ & 28.3447 & & 28.4573 & \\
\hline \multirow{4}{*}{2} & $\rho$ & 0.3669 & & $1 \& 0.3683$ & \\
\hline & $b_{1}$ & 0.0003 & 1.4266 & 0.0003 & 1.4249 \\
\hline & $b_{2}$ & 58.9057 & & 58.8892 & \\
\hline & $u$ & 30.6943 & & 30.7584 & \\
\hline \multirow{4}{*}{3} & $\rho$ & 0.4193 & & $1 \& 0.4384$ & \\
\hline & $b_{1}$ & 0.0025 & 0.9756 & 0.0023 & 0.9588 \\
\hline & $b_{2}$ & 62.1686 & & 61.9513 & \\
\hline & $u$ & 37.5064 & & 38.5224 & \\
\hline
\end{tabular}

\subsection{Optimal GMC(1,n)}

To improve the accuracy of the predictive model $\operatorname{GMC}(1, \mathrm{n})$ instead of the weighted mean to determine the grey derivative, we introduce the coefficient $\rho \in[0 ; 1]$, then Equation (7) takes the following form:

$$
B=\left[\begin{array}{lll}
Q_{Y}(1) & Q_{X}(1) & 1 \\
Q_{Y}(2) & Q_{X}(2) & 1 \\
Q_{Y}(r-1) & \left(Q_{X}(r-1)\right. & 1
\end{array}\right]
$$

where

$$
\begin{gathered}
Q_{Y}(r)=-(1-\rho) Y^{(1)}(r)+\rho Y^{(1)}(r+1) \\
Q_{X}(r)=(1-\rho) X^{(1)}(r)+\rho X^{(1)}(r+1)
\end{gathered}
$$

Coefficient $\rho$ is determined by a PSO method [26]. This algorithm is a system of particles that move to optimal solutions, each particle contains the coordinates of the found best solution (pbest) and the best solution from all the particles in the swarm (gbest). The direction and length of the particle velocity vector is determined by the following formula:

$$
v_{i}=v_{i}+a_{1} \text { rnd }() \cdot\left(\text { pbest }_{i}-x_{i}\right)+a_{2} \text { rnd }() \cdot\left(\text { gbest }_{i}-x_{i}\right)
$$

where $v_{i}$ is the particle speed vector, $a_{1}, a_{2}$ are the constant accelerations and $x$ is the particle current position. In this case the particle current position is the $\rho$. As an optimality criterion, the minimum of Equation (16) is used. The obtained predictive models and the RMSPE are presented in Table 2, where it can be seen that the accuracy of the grey model is improved.

In [25], interpolation coefficients $\rho_{i}$ are introduced into the background values of each of the variables in $\operatorname{GDMC}(1, \mathrm{n})$. Application of this algorithm to $\operatorname{GMC}(1, \mathrm{n})$ model will have the following changes in Equation (7):

$$
B=\left[\begin{array}{lll}
Q_{Y 1}(1) & Q_{X 2}(1) & 1 \\
Q_{Y 1}(2) & Q_{X 2}(2) & 1 \\
Q_{Y 1}(r-1) & \left(Q_{X 2}(r-1)\right. & 1
\end{array}\right]
$$

where

$$
\begin{gathered}
Q_{Y i}(r)=-\left(1-\rho_{i}\right) Y^{(1)}(r)+\rho_{i} Y^{(1)}(r+1) \\
Q_{X i}(r)=\left(1-\rho_{i}\right) X^{(1)}(r)+\rho_{i} X^{(1)}(r+1)
\end{gathered}
$$

The predictive results have changed slightly (Table 2 ), but the time to find the optimal values of the $\rho_{i}$ increased. Increase of $(n-1)$ dependent variables leads to increased optimization time exponentially and to a difficulty in using the algorithm with different coefficients for each variable in real-time control. Therefore to enhance the modelling and predictive accuracy one coefficient $\rho$ is used to control the task. The coefficients of the grey model (Table 2), which were found by Equation (6), differ significantly for each sample. This is due to the fact that each temperature curve was obtained under different initial conditions. Moreover, in production under real conditions, it is not possible to continuously control the composition of the charge. Therefore, it is necessary to build a predictive model, which uses only measured real time factors and dynamically constructs the model for a certain batch of sintered ore.

\section{4 | CONTROL STRUCTURE}

Dynamic construction of the grey model uses only actual data, which represents real time information about the sintering process, and does not depend on historical data, 


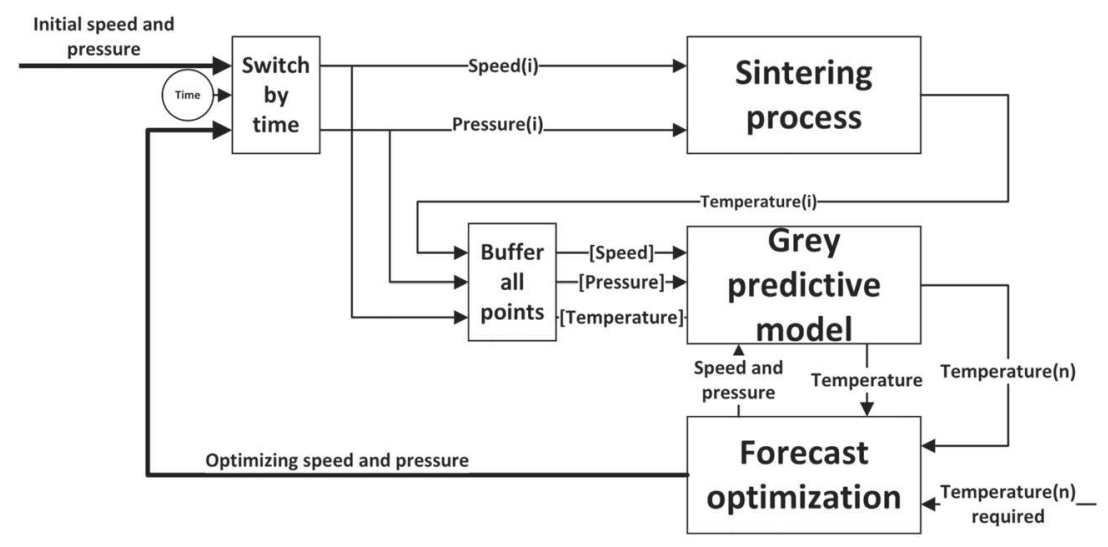

making it possible to obtain the prediction of the charge temperature. If the predictive temperature of the charge at the end of the strand does not reach the set point (the highest temperature in the wind boxes), then it is necessary to control the sinter process. The control can be carried out by changing the amount of fuel in the initial charge, the strand speed and the underpressure created in the wind boxes. The change in the amount of fuel affects only the charge that has not yet been fed to the sinter, so this effect is not considered in the work.

The idea to build the prediction model of the BTP in real time was also considered in [16], where the model parameters are determined by the closed-loop identification method. High accuracy of the generalized prediction model [16] was achieved by a real-time measurement of additional parameters, which makes the proposed model more expensive.

The proposed control structure for the BTP is presented in Figure 6 and has the following algorithm: after the charge is passed under the horn, the system begins collecting data on temperature, pressure in the wind boxes, and sinter machine speed. Based on the collected data, a forecast model is built on the basis of the grey model that predicts the temperature at the end of the sintering machine. If the temperature does not correspond to the BTP, a predictive optimization algorithm calculates the necessary values of the sinter velocity and pressure in the wind boxes. The resulting values are fed to the appropriate controllers. After the charge reaches the end of the sintering machine, the cycle is repeated for the next charge.

The PSO is used as a method of predictive optimization. Its distinguishing feature from many other methods is that, for the particle swarm method, we can calculate only the value of the function being optimized, but not its gradient. In terms of efficiency, it can compete with other methods of global optimization, and its low algorithmic complexity contributes to the simplicity of its implementation. Particle current position Equation (18) includes two variables: number of samples that represents time of sinter process and gas velocity.
The PSO algorithm is composed of the following steps.

1. Generate $m$-particles $(m=2)$ in the $n$-dimensional space. Position and velocity are represented by $x_{i}=$ $\left(x_{i 1}, x_{i 2}, . ., x_{i n}\right)$ and $v_{i}=\left(v_{i 1}, v_{i 2}, . ., v_{i n}\right), i=1, . ., m$.

2. Generate randomly the initial position of the particles $x_{i}$ within the limits $t_{\min } \leqslant x_{1} \leqslant t_{\max }, X_{\min } \leqslant x_{2} \leqslant X_{\max }$ and initial velocity vector $v_{i} . t$ is the time step and $X$ is the underpressure in the wind boxes.

3. According to Equation (6) substitute $x_{2}$ into the matrix $B$ and according to Equation (9) put $x_{1}$ as parameter $t$.

4. Calculate the fitness value Equation (16) for each particle.

5. Compare each particle's fitness to the global best position pbest to adapt to the value; if it is the optimum, it can be taken as the best position; if not, turn to next step.

6. Update particles velocity and position according to the evolution Equation (18).

7. If stopping criteria are met, show the output and its fitness, which corresponds to the optimal parameters and the RMSPE, or else go back to step 3.

With the help of the obtained optimal sintering process time $t$ value, the strand speed is determined, and through the optimum gas velocity value, the underpressure in wind boxes is found according to Equation (1). Modeling results using the BTP control system for the samples are represented in Figure 7. In the first sample, the sintering temperature of the charge is reached in $22 \mathrm{~min}$ utes. To increase line productivity, extended boundaries of inequalities are set,wherein, the optimization algorithm allows to find such values of strand speed and pressure in wind boxes at which the BTP is reached in $19.5 \mathrm{~min}$ utes. Sample 1 results of the forecast optimization are as follows: the speed values change from $3.5 \mathrm{~m} / \mathrm{min}$ to 4.5 $\mathrm{m} / \mathrm{min}$ and the pressure in wind boxes from $800 \mathrm{mmHg}$ to $760 \mathrm{mmHg}$. In the second sample, the BTP is not reached. Therefore, as a result of predictive optimization, a new value is supplied to the pressure regulator, at which the sintering temperature is reached. In the third case, as a result of predictive optimization, the speed of sin- 

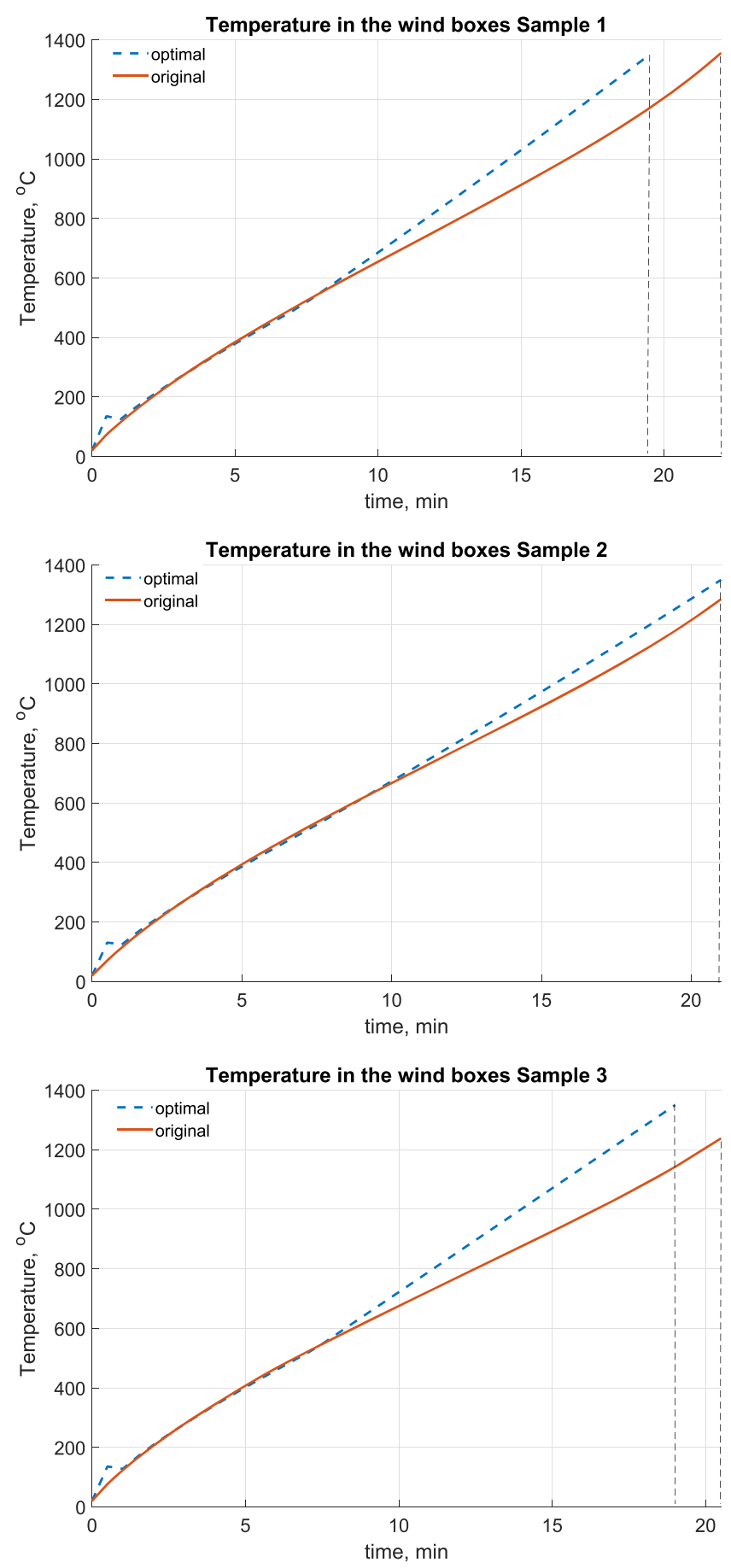

FIGURE 7 Modeling results of the BTP using the control structure [Color figure can be viewed at wileyonlinelibrary.com]

ter strand and pressure in wind boxes is increased, which allows for achievement of the optimum temperature in $19 \mathrm{~min}$.

\section{CONCLUSIONS}

The proposed control process structure of phosphorite ore sintering considered in this paper is intended to improve the product quality and reduce the return. As an indicator responsible for sintering quality, the BTP was cho- sen, that is, the position at which the process reaches the highest temperature. Prediction of this variable was realized through the grey predictive model with some main contributions: (1) dynamic construction of the predictive model using a small sample size, obtained from the beginning of the process, which reduces the time for data acquisition and system training; (2) control of the sintering process not only based on the strand speed, but also on the underpressure in the wind boxes; (3) use of a grey model with (n-1) influencing factors that takes into account not only the effect of the predicted value, but also other process variables; (4) modeling different continuous grey models and development of optimal $\operatorname{GMC}(1, \mathrm{n})$ model; (5) use of predictive optimization algorithm to determine optimal process parameters. In the framework of this article, the initial amount of data for constructing the model was also determined, as a result of which the amount of costs for building the data acquisition system is reduced, while the predictive model error does not exceed $1.5 \%$.

\section{ORCID}

Hassen Fourati iD https://orcid.org/0000-0002-8398-7183

\section{REFERENCES}

1. L. Peng, Z. Ji, and J. Tan, Sintering finish point intelligent control, Proceedings of 2005 IEEE/ASME International Conference on Advanced Intelligent Mechatronics, Monterey, CA, USA, 2006.

2. S. Du et al., Intelligent coordinating control between burn-through point and mixture bunker level in an iron ore sintering process, J. Adv. Comput. Int. Int. Inf. 21 (2017), 140-147.

3. J. Wang et al., BTP prediction of sintering process by using multiple models, 26th Chinese Control and Decision Conference (CCDC 2014), Vol. 2014, Changsha, China, 2014.

4. M. Wu, C. H. Xu, and Y. X. Du, Intelligent optimal control for lead-zinc sintering process state, Trans. Nonferrous Metals Soc. China 16 (2006), 975-981.

5. M. Wu et al., Neural-network-based integrated model for predicting burn-through point in lead-zinc sintering process, J. Process. Control. 22 (2012), 925-934.

6. M. Wu et al., An intelligent control system based on prediction of the burn-through point for the sintering process of an iron and steel plant, Expert Syst. Appl. 39 (2012), 5971-5981.

7. S. Du et al., A fuzzy control strategy of burn-through point based on the feature extraction of time series trend for iron ore sintering process, IEEE Trans. Ind. Inform. (2019), 1-9.

8. X. Chen et al., T-S Fuzzy logic based modeling and robust control for burning-through point in sintering process, IEEE Trans. Ind. Electron. 64 (2017), 9378-9388.

9. W. S. Cheng, An application of adaptive genetic-neural algorithm to sinter's BTP process, IEEE International Conference on Machine Learning and Cybernetics, Shanghai, China, 2005, pp. 3356-3360.

10. W. S. Cheng, Prediction system of burning through point (BTP) based on adaptive pattern clustering and feature map, International Conference on Machine Learning and Cybernetics, Dalian,China, 2006, pp. 3089-3094. 
11. X. Q. Shang et al., Data-driven prediction of sintering burn-through point based on novel genetic programming, J. Iron. Steel Res. Int. 17 (2010), 1-10.

12. D. Wang et al., Application research based on GA-FWA in prediction of sintering burning through point, International Conference on Computer, Communications and Mechatronics Engineering, 2018, pp. 378-385.

13. B. Kim et al., Estimation of burn-through point in the sinter process, 14th International Conference on Control, Automation and Systems, Seoul, Korea, 2014, pp. 1531-1533.

14. C. S. Wang and M. Wu, Hierarchical intelligent control system and its application to the sintering process, IEEE Trans. Ind. Inf. 9 (2013), 190-196.

15. W. Cao et al., A dynamic subspace model for predicting burn-through point in iron sintering process, Inform. Sci. (2018), $1-12$.

16. M. Wu et al., Design and application of generalized predictive control strategy with closed-loop identification for burn-through point in sintering process, Control. Eng. Practice 20 (2012), 1065-1074.

17. J. Shi et al., Soft sensing of the burning through point in iron-making process, IEEE International Conference on Cognitive Informatics \& Cognitive Computing (ICCI*CC 2016), Palo Alto, CA, USA, 2016.

18. S. Du et al., Intelligent integrated control for burn-through point to carbon efficiency optimization in iron ore sintering process, IEEE Trans. Control Syst. Technol. (2019).

19. J. Deng, Introduction to grey system, J. Grey Syst. 1 (1989), 1-24.

20. T. L. Tien, The indirect measurement of tensile strength of material by the grey prediction model $\operatorname{GMC}(1, n)$, Meas. Sci. Technol. 16 (2005), 1322-1328.

21. Y. Kaymak, T. Hauck, and M. Hillers, Iron ore sintering process model to study local permeability control, COMSOL Conference, Rotterdam, Netherlands, 2017.

22. T. L. Tien, The indirect measurement of tensile strength for a higher temperature by the new model $\operatorname{IGDMC}(1, n)$, Measurement, J. Int Meas. Conf. 41 (2008), 662-675.

23. T. L. Tien, The indirect measurement of tensile strength by the new model FGMC (1, n), Measurement, J. Int. Meas. Conf. 44 (2011), 1884-1897.

24. T. L. Tien, The deterministic grey dynamic model with convolution integral DGDMC(1, n), Appl. Math. Model. 33 (2009), 3498-3510.

25. Z. X. Wang and L. L. Pei, An optimized grey dynamic model for forecasting the output of high-tech industry in China, Math Probl. Eng. (2014).

26. J. Kennedy and R. Eberhart, Particle swarm optimization, Neural Networks, 1995.

\section{AUTHOR BIOGRAPHIES}

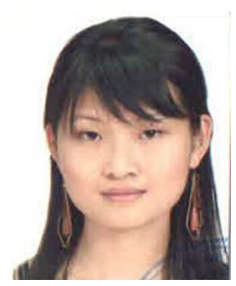

Nigina Toktassynova is currently a Ph.D. student of automation and control at the Satbayev University, Almaty, Kazakhstan. She earned her bachelor and master's degree in automation and control at the Almaty University of Power Engi- neering and Telecommunication, Almaty, Kazakhstan, in 2012, and 2014, respectively. Her research interests include predicitive algorithms, grey system theory, and control systems in sintering process.

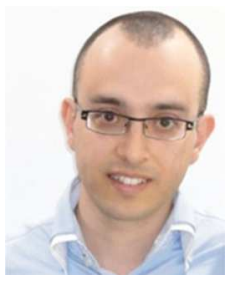

Hassen Fourati is currently an associate professor of electrical engineering and computer science at the University of Grenoble Alpes, Grenoble, France, and a member of the Dynamics and Control of Networks Team (DANCE), affiliated with the Pôle Automatique et Diagnostic of the GIPSA-Lab. He earned his bachelor of engineering degree in electrical engineering at the National Engineering School of Sfax, Tunisia; master's degree in automated systems and control at the University of Claude Bernard, Lyon, France; and Ph.D. in automatic control at the University of Strasbourg, France, in 2006, 2007, and 2010, respectively. His research interests include nonlinear filtering, estimation, and multisensor fusion with applications in navigation, motion analysis, mobility, and traffic management.

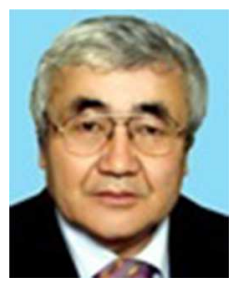

Batyrbek Suleimenov is currently a professor and Head of the Automation and Control Department at Satbayev University, Almaty, Kazakhstan. He is a member of the International Engineering Academy of Kazakhstan. He has 6 patents and more than 200 publications. His research interests include intelligent control systems for technological processes and systems for operational diagnostics of technological equipment.

How to cite this article: Toktassynova N, Fourati H, Suleimenov B. Application of grey system theory to phosphorite sinter process: From modeling to control. Asian J Control. 2020;1-10. https://doi.org/10.1002/asjc.2348 